\title{
ASYMPTOTIC ARBITRAGE IN THE HESTON MODEL
}

\author{
FATMA HABA \\ Department of Mathematics \\ Université Tunis El Manar, Tunisia \\ haba_fatma@hotmail.fr \\ ANTOINE JACQUIER \\ Department of Mathematics, Imperial College London \\ South Kensington Campus, London SW7 2AZ, UK \\ a.jacquier@imperial.ac.uk \\ Received 7 May 2014 \\ Accepted 31 August 2015 \\ Published 30 November 2015
}

\begin{abstract}
In this paper, we introduce a new form of asymptotic arbitrage, which we call a partial asymptotic arbitrage, half-way between those of Föllmer \& Schachermayer (2007) [Mathematics and Financial Economics 1 (34), 213-249] and Kabanov \& Kramkov (1998) [Finance and Stochastics 2, 143-172]. In the context of the Heston model, we establish a precise link between the set of equivalent martingale measures, the ergodicity of the underlying variance process and this partial asymptotic arbitrage. In contrast to Föllmer \& Schachermayer (2007) [Mathematics and Financial Economics 1 (34), 213-249], our result does not assume a suitable condition on the stock price process to allow for (partial) asymptotic arbitrage.
\end{abstract}

Keywords: Stochastic volatility model; Heston model; asymptotic arbitrage; large deviations.

\section{Introduction}

The concept of arbitrage is the cornerstone of modern mathematical finance, and several versions of the so-called fundamental theorem of asset pricing have been proved over the past two decades, see for instance Delbaen \& Schachermayer (2006) for an overview. A version of it essentially states that absence of arbitrage is equivalent to the existence of an equivalent martingale measure under which discounted asset prices are true martingales. This then allows the use of "martingale models" (either continuous or with jumps) as underlying dynamics for option pricing. In practice, should short-term arbitrages arise — due to some market

This is an Open Access article published by World Scientific Publishing Company. It is distributed under the terms of the Creative Commons Attribution 4.0 (CC-BY) License. Further distribution of this work is permitted, provided the original work is properly cited. 
discrepancies - they are immediately exploited by traders, and market liquidity therefore acts as an equilibrium agent, to prevent them from occurring significantly. It can be argued, however, that one may generate long-term riskless profit, when the time horizon tends to infinity. This turns out to be the case in most models used in practice, and the existence and nature of such infinite horizon asymptotic arbitrage opportunities have been studied by Bidima \& Rasonyi (2015), Cordero \& PerezOstafe (2015), Du \& Neufeld (2013), Klein et al. (2014), Klein \& Schachermayer (1996) and Rokhlin (2008).

Among the plethora of models used and analyzed both in practice and in theory, stochastic volatility models have proved to be very flexible and suitable for pricing and hedging. Due to its affine structure, the Heston (1993) model has gained great popularity among practitioners for equity and FX derivatives modeling (see Fouque et al. (2011) and Gatheral (2006) for a detailed account). Because of the correlation between the asset price and the underlying volatility, the market is incomplete, and the Heston model admits an infinity of equivalent martingale measures. Its affine structure allows us to study precisely the existence (or absence) of asymptotic arbitrage. Specifically, we shall endeavor to understand how the parameters of the model influence the nature - such as its speed and existence - of this asymptotic arbitrage. Of particular interest will be the link between asymptotic arbitrage and the ergodicity of the underlying variance process. In Föllmer \& Schachermayer (2007), the authors proved under suitable regularity conditions that price processes with a nontrivial market price of risk (see Definition 2.4) allow for asymptotic arbitrage (with linear speed). We consider a real-valued semi-martingale $S=\left(S_{t}\right)_{t \geq 0}$, modeling the price process of a risky asset under an equivalent martingale measure with infinite horizon. In this paper, We introduce a new and intermediate form of asymptotic arbitrage between the two forms recalled from Föllmer \& Schachermayer (2007) and Kabanov \& Kramkov (1998), called a partial asymptotic arbitrage. Using the theory of large deviations, we shall show that $S$, may allow for such new form of asymptotic arbitrage even if it does not admit an average squared market price of risk.

The organization of this paper is as follows: all the notations and definitions are given in Sec. 2. Partial asymptotic arbitrage in the Heston model is studied in Sec. 3; the main contribution of this paper is Theorem 3.1, which identifies sufficient (and sometimes necessary) conditions on the set of equivalent martingale measures under which partial asymptotic arbitrage occurs with linear speed. These conditions are different from those in Proposition 3.4 in which we study the role of the ergodicity of the variance process on the existence of partial asymptotic arbitrage with slower speed.

\section{Notations and Definitions}

Let $(\Omega, \mathcal{F}, \mathbb{F}, \mathbb{P})$ be a filtered probability space where the filtration $\mathbb{F}=\left(\mathcal{F}_{t}\right)_{t \geq 0}$ satisfies the usual conditions, and let $S$ denote a stochastic stock price process adapted 
to $\mathbb{F}$. We let $\mathcal{H}$ denote the class of predictable, $S$-integrable processes that are admissible in the sense of Definition 2.1.4 in Delbaen \& Schachermayer (2006), and define the sets of attainable strategies $\mathcal{K}_{t}$ and of equivalent local martingales $\mathcal{M}_{t}^{e}(S)$ by

$$
\begin{aligned}
\mathcal{K}_{t} & :=\left\{\int_{0}^{t} H_{u} d S_{u}: H \in \mathcal{H}\right\} \cap L^{0}(\Omega, \mathcal{F}, \mathbb{F}, \mathbb{P}), \\
\mathcal{M}_{t}^{e}(S): & =\left\{\mathbb{Q} \sim \mathbb{P}:\left(S_{u}\right)_{0 \leq u \leq t} \text { is a local } \mathbb{Q} \text {-martingale }\right\},
\end{aligned}
$$

where $L^{0}(\Omega, \mathcal{F}, \mathbb{F}, \mathbb{P})$ denotes the space of all $\mathcal{F}$-measurable real-valued functions. We assume that $\mathcal{M}_{t}^{e}(S)$ is not empty and, for any set $A$ in $\Omega$, we denote its complement $A^{c}:=\Omega \backslash A$. We always assume here that the financial market satisfies the no-arbitrage condition, as defined in Definition 2.2.3 in Delbaen \& Schachermayer (2006) as a condition on the sets $\mathcal{K}_{t}$ (for any $t \geq 0$ ) and on the cone of contingent claims that are super-replicable at inception.

\subsection{Asymptotic arbitrage}

We are interested here in specific forms of arbitrage (asymptotic arbitrage), first introduced by Kabanov \& Kramkov (1998), and refined recently by Definition 1.1 in Föllmer \& Schachermayer (2007). The following definition of an $\left(\varepsilon_{1}, \varepsilon_{2}\right)$-arbitrage is taken from the latter (Proposition 2.1 in Föllmer \& Schachermayer (2007)):

Definition 2.1. Let $\left(\varepsilon_{1}, \varepsilon_{2}\right) \in(0,1)^{2}$. The process $S$ admits an $\left(\varepsilon_{1}, \varepsilon_{2}\right)$-arbitrage (up to time $T>0$ ) if there exists $X_{T} \in \mathcal{K}_{T}$ such that

(1) $X_{T} \geq-\varepsilon_{2} \mathbb{P}$-almost surely;

(2) $\mathbb{P}\left(X_{T} \geq 1-\varepsilon_{2}\right) \geq 1-\varepsilon_{1}$.

This means that the maximal loss of the trading strategy, yielding the wealth $X_{T}$ at time $T$, is bounded by $\varepsilon_{2}$ and, with probability $1-\varepsilon_{1}$, the terminal wealth $X_{T}$ equals at least $1-\varepsilon_{2}$. Let us consider the following slightly weaker version, which imposes less stringent restrictions on the maximal loss:

Definition 2.2. Let $\left(e_{1}, e_{2}\right) \in[0,1)^{2}$. We say that the process $S$ admits a partial $\left(e_{1}, e_{2}\right)$-arbitrage (up to time $\left.T>0\right)$ if for any $\left(\varepsilon_{1}, \varepsilon_{2}\right) \in\left(e_{1}, 1\right) \times\left(e_{2}, 1\right)$, there exists $X_{T} \in \mathcal{K}_{T}$ such that

(1) $X_{T} \geq-\varepsilon_{2} \mathbb{P}$-almost surely;

(2) $\mathbb{P}\left(X_{T} \geq 1-\varepsilon_{2}\right) \geq 1-\varepsilon_{1}$.

Obviously, Definition 2.2 is equivalent to Definition 2.1 when $e_{1}=e_{2}=0$. However, as we shall see later, (partial) asymptotic arbitrage may only appear for $e_{1}$ exponentially small. In that case, this alternative definition may also be seen as a mid-point characterization between Definition 2.1 and Definition 2.3 below. The latter in particular characterizes the notion of asymptotic exponential arbitrage with exponentially decaying failure probability, first proposed by Föllmer \& Schachermayer (2007) and studied later by Bidima \& Rasonyi (2012) and Du \& Neufeld 
(2013). In the context of stochastic volatility models, see Sec. 2.2 below, Föllmer \& Schachermayer (2007) introduced a condition on the market price of risk (or equivalently on the set of equivalent probability measures in an incomplete market) which is sufficient for asymptotic arbitrage (in the sense of Definition 2.1). As we shall see below for the Heston model, it is however not necessary, and, when violated, partial asymptotic arbitrage (Definition 2.2) may still occur.

Definition 2.3. The process $S$ allows for asymptotic exponential arbitrage with exponentially decaying failure probability if there exist $t_{0} \in(0, \infty)$ and constants $C, \lambda_{1}, \lambda_{2}>0$ such that for all $t \geq t_{0}$, there is $X_{t} \in \mathcal{K}_{t}$ satisfying

(1) $X_{t} \geq-\mathrm{e}^{-\lambda_{2} t} \mathbb{P}$-almost surely;

(2) $\mathbb{P}\left(X_{t} \leq \mathrm{e}^{\lambda_{2} t}\right) \leq C \mathrm{e}^{-\lambda_{1} t}$.

We see that there is a relation between Definitions 2.3 and 2.2. If the process $S$ allows for asymptotic exponential arbitrage with exponentially decaying failure probability, then it admits a partial $\left(e_{1}, e_{2}\right)$-arbitrage with $e_{1}=C \mathrm{e}^{-\lambda_{1} t}$ and $e_{2}=\mathrm{e}^{-\lambda_{2} t}$. Indeed, for any $\varepsilon_{2} \in\left(\mathrm{e}^{-\lambda_{2} t}, 1\right)$ we have $X_{t} \geq-\mathrm{e}^{-\lambda_{2} t} \geq-\varepsilon_{2}$. Now for large $t$, we have $1-C \mathrm{e}^{-\lambda_{1} t} \leq \mathbb{P}\left(X_{t}>\mathrm{e}^{\lambda_{2} t}\right) \leq \mathbb{P}\left(X_{t} \geq 1-\mathrm{e}^{-\lambda_{2} t}\right)=\mathbb{P}\left(X_{t} \geq 1-\varepsilon_{2}\right)$. Then for any $\varepsilon_{1} \in\left(C \mathrm{e}^{-\lambda_{1} t}, 1\right)$ with $C \in(0,1)$, we have $\mathbb{P}\left(X_{t} \geq 1-\varepsilon_{2}\right) \geq 1-\varepsilon_{1}$.

Kabanov \& Kramkov (1998), related (strong) asymptotic arbitrage (essentially in the sense of Definition 2.1) to the (Hellinger) distance of the set of equivalent martingale measures to the original measure. We follow here the approach of Föllmer \& Schachermayer (2007), where the connection is made at the level of utility functions. For any $x, T \geq 0$, define the value function

$$
u_{T}(x):=\sup _{X_{T} \in \mathcal{K}_{T}} \mathbb{E}\left(U\left(x+X_{T}\right)\right),
$$

where $U:[0, \infty) \rightarrow \mathbb{R}$ is any strictly increasing concave function satisfying $\lim _{x \downarrow 0} U(x)=-\infty$ and $\lim _{x \uparrow \infty} U(x)=0$. Then the following holds:

Proposition 2.1. Let $\left(e_{1}, e_{2}\right) \in[0,1)^{2}$ and $\left(\varepsilon_{1}, \varepsilon_{2}\right) \in\left(e_{1}, 1\right) \times\left(e_{2}, 1\right)$. For any $\alpha \in(-\infty, 0)$, let $\varepsilon \leq \varepsilon_{1} \varepsilon_{2}^{|\alpha|}$. If $u_{T}(x) \geq \varepsilon x^{\alpha} / \alpha$ for all $x>0$, then $X$ admits $a$ partial $\left(e_{1}, e_{2}\right)$ arbitrage.

Proof. The proof is the same as that of Proposition 2.3 (part d) in Föllmer \& Schachermayer (2007). The reverse, however, does not necessarily hold here. In the proof by Föllmer and Schachermayer, $\varepsilon_{1}$ and $\varepsilon_{2}$ are required to be taken as small as possible. This is not feasible here because of the $\left(e_{1}, e_{2}\right)$ lower bound we impose in our definition of partial asymptotic arbitrage (see also Theorem 3.1 below for a precise example in the Heston framework).

\subsection{Stochastic volatility models}

We consider here the Heston stochastic volatility model, namely the unique strong solution to the stochastic differential equations (2.1) below. As is well known 
(Heyde \& Wong 2006), there may not be a unique risk-neutral martingale measure for this. The following SDEs are therefore understood under one such risk-neutral measure $\mathbb{Q}$.

$$
\begin{aligned}
d S_{t} / S_{t} & =\mu d t+\sqrt{V_{t}}\left(\rho d W_{1}(t)+\sqrt{1-\rho^{2}} d W_{2}(t)\right), \quad S_{0}=1, \\
d V_{t} & =\left(a-b V_{t}\right) d t+\sqrt{2 \sigma V_{t}} d W_{1}(t), \quad V_{0}>0
\end{aligned}
$$

where $W_{1}$ and $W_{2}$ are independent $\mathbb{Q}$-Brownian motions, $a, \sigma>0, \mu, b \in \mathbb{R}$ and $|\rho|<1$. The class of equivalent martingale measures $\mathbb{Q}$ can be considered in terms of the Radon-Nikodym derivatives

$$
\begin{aligned}
Z_{t}:=\left.\frac{d \mathbb{Q}}{d \mathbb{P}}\right|_{\mathcal{F}_{t}}= & \exp \left\{-\left(\int_{0}^{t} \gamma_{1}(s) d W_{1}(s)+\int_{0}^{t} \gamma_{2}(s) d W_{2}(s)\right)\right. \\
& \left.-\frac{1}{2}\left(\int_{0}^{t} \gamma_{1}^{2}(s) d s+\int_{0}^{t} \gamma_{2}^{2}(s) d s\right)\right\},
\end{aligned}
$$

where $\gamma_{i}$ are the market price of risk processes corresponding to $W_{i}$, for $i=1,2$. The condition $\mu-r=\sqrt{V_{t}}\left(\rho \gamma_{1}(t)+\sqrt{1-\rho^{2}} \gamma_{2}(t)\right)$ is necessary for an equivalent local martingale measure to exist, and ensures that the discounted stock price is a local martingale; here $r$ denotes the constant risk-free rate. Since $Z$ is a positive local martingale with $Z_{0}=1$, it is a supermartingale, and a true martingale if and only if $\mathbb{E}\left(Z_{t}\right)=1$. For the Heston stochastic volatility model we obtain, for any $\lambda \in \mathbb{R}$

$$
\gamma_{1}(t)=\lambda \sqrt{V_{t}} \quad \text { and } \quad \gamma_{2}(t)=\frac{1}{\sqrt{1-\rho^{2}}}\left(\frac{\mu-r}{\sqrt{V_{t}}}-\lambda \rho \sqrt{V_{t}}\right)
$$

Definition 2.4. Let $f: \mathbb{R}_{+}^{*} \rightarrow \mathbb{R}_{+}^{*}$ be a smooth function such that $\lim _{t \uparrow \infty} f(t)=$ $+\infty$. The process $S$ is said to have an average squared market price of risk $\gamma$ above the threshold $c>0$ with speed $f(t)$ if $\mathbb{P}\left(f(t)^{-1} \int_{0}^{t} \gamma^{2}(s) d s<c\right)$ tends to zero as $t$ tends to infinity.

\section{Main Results}

For any $(\alpha, \beta, \delta) \in \mathbb{R}^{3}$, we introduce the process $\left(X_{t}^{\alpha, \beta, \delta}\right)_{t \geq 0}$ defined (pathwise) by

$$
X_{t}^{\alpha, \beta, \delta}:=\alpha V_{t}+\beta \int_{0}^{t} V_{s} d s+\delta \int_{0}^{t} V_{s}^{-1} d s, \quad \text { for any } t \geq 0,
$$

where $V$ is the Feller diffusion for the variance in (2.1). We shall always assume that $\beta$ and $\delta$ are not both null simultaneously. In that case, $X^{\alpha, \beta, \delta}$ is simply the Feller diffusion, and its density is known in closed form (see Part 1, Chapter 6.3 in Jeanblanc et al. (2009)). The large-time behavior of $X^{\alpha, \beta, \delta}$ will play a key role in determining average squared market prices of risk, and the case $\beta=\delta=0$ will 
never occur, so this assumption does not entail any loss of generality here. Define the real interval $\mathcal{D}_{\beta, \delta}$ by

$$
\mathcal{D}_{\beta, \delta}= \begin{cases}{\left[\frac{(a-\sigma)^{2}}{4 \sigma \delta}, \frac{b^{2}}{4 \sigma \beta}\right],} & \text { if } \beta>0, \delta<0, \\ \left(-\infty, \frac{(a-\sigma)^{2}}{4 \sigma \delta} \wedge \frac{b^{2}}{4 \sigma \beta}\right], & \text { if } \beta>0, \delta>0, \\ {\left[\frac{b^{2}}{4 \sigma \beta}, \frac{(a-\sigma)^{2}}{4 \sigma \delta}\right],} & \text { if } \beta<0, \delta>0, \\ {\left[\frac{(a-\sigma)^{2}}{4 \sigma \delta} \vee \frac{b^{2}}{4 \sigma \beta},+\infty\right),} & \text { if } \beta<0, \delta<0 .\end{cases}
$$

Whenever $\beta \delta=0$, we define $\mathcal{D}_{\beta, \delta}$ by taking the limits of the interval (a closed bound becoming open if it becomes infinite), where we use the slight abuse of notation " $1 / 0=\infty$ ", i.e. $\mathcal{D}_{\beta, \delta}=\left(-\infty, \frac{b^{2}}{4 \sigma \beta}\right]$ if $\beta>0$ and $\delta=0, \mathcal{D}_{\beta, \delta}=\left[\frac{b^{2}}{4 \sigma \beta},+\infty\right)$ if $\beta<0$ and $\delta=0$. Let us further define the function $\Lambda^{\beta, \delta}: \mathcal{D}_{\beta, \delta} \rightarrow \mathbb{R}$ by

$$
\Lambda^{\beta, \delta}(u)= \begin{cases}\frac{b a}{2 \sigma}-\frac{1}{2 \sigma} \sqrt{\left((a-\sigma)^{2}-4 \sigma \delta u\right)\left(b^{2}-4 \sigma \beta u\right)}-\frac{1}{2} \sqrt{b^{2}-4 \sigma \beta u}, & \text { if } \delta \neq 0, \\ \frac{a}{2 \sigma}\left(b-\sqrt{b^{2}-4 \sigma \beta u}\right), & \text { if } \delta=0 .\end{cases}
$$

In the case $\delta \neq 0$ above, we further impose the condition $a>\sigma$ for the definition of the function $\Lambda^{\beta, \delta}$.

Remark 3.1. It may be surprising at first that the function $\Lambda^{\beta, \delta}$ does not depend on $\alpha$. This function actually describes the large-time behavior of the process $X^{\alpha, \beta, \delta}$. Since the variance process $V$ is strictly positive almost surely (by the Feller condition imposed above), the term $\int_{0}^{t} V_{s} d s$ clearly dominates $V_{t}$ for any $t$, which explains why $\alpha$ bears no influence on $\Lambda^{\beta, \delta}$. The condition $a>\sigma$ imposed above in the case $\delta \neq 0$ should not surprise the reader since this is nothing else than the Feller condition, ensuring that the variance process never touches the origin almost surely.

We further define the Fenchel-Legendre transform $\Lambda_{\beta, \delta}^{*}: \mathbb{R} \rightarrow \mathbb{R}_{+}$of $\Lambda^{\beta, \delta}$ by

$$
\Lambda_{\beta, \delta}^{*}(x):=\sup _{u \in \mathcal{D}_{\beta, \delta}}\left\{u x-\Lambda^{\beta, \delta}(u)\right\}
$$

Notation 3.1. Whenever $\beta=0$ or $\delta=0$, we shall drop the subscript and write respectively $\Lambda^{\delta}$ or $\Lambda^{\beta}$. The same rule shall apply for the Fenchel-Legendre transforms and their respective domains.

In general, $\Lambda_{\beta, \delta}^{*}$ does not have a closed-form representation. However when $\delta$ is null — which shall be of interest for us — it actually does, and a straightforward 
computation shows that

$$
\Lambda_{\beta, 0}^{*}(x) \equiv \Lambda_{\beta}^{*}(x)=\frac{(b x-a \beta)^{2}}{4 \sigma|\beta x|}, \quad \text { for all } x \in \mathbb{R}^{*} .
$$

In that case, the function $\Lambda_{\beta}^{*}$ is strictly convex on $\mathbb{R}_{+}^{*}$ (respectively on $\mathbb{R}_{-}^{*}$ ) with a unique minimum attained at $|a \beta / b|$ (respectively at $-|a \beta / b|$ ). In particular, if $b \beta>0$, then $\Lambda_{\beta}^{*}(|a \beta / b|)=0$ and $\Lambda_{\beta}^{*}(x)>0$ for all $x \in \mathbb{R}_{+}^{*} \backslash\{|a \beta / b|\}$. Symmetric statements hold on $\mathbb{R}_{-}$.

\subsection{The large deviations case}

In this section, we prove asymptotic arbitrage results (with linear speed) for the stock price process; we shall in particular observe that the ergodicity of the variance process plays a key role. We first start with the following lemma (proved in Appendix A), which will be used heavily in the remaining of the paper. For precise definitions of large deviations principles (LDP), we refer the reader to the excellent monograph by Dembo \& Zeitouni (1993); we shall use the nonstandard terminology "partial large deviations principles" if an LDP holds only on subsets of the real line.

Lemma 3.1. As $t$ tends to infinity, the family $\left(t^{-1} X_{t}^{\alpha, \beta, \delta}\right)_{t \geq 0}$ satisfies

(1) a full $L D P($ on $\mathbb{R})$ if $\beta \delta<0$;

(2) a partial $L D P$ on $(2 \sqrt{\delta \beta},+\infty)$ if $\beta \geq 0$ and $\delta \geq 0$;

(3) a partial LDP on $(-\infty,-2 \sqrt{\delta \beta})$ if $\beta \leq 0$ and $\delta \leq 0$;

In each case, the rate function is $\Lambda_{\beta, \delta}^{*}$ and the (partial) LDP holds with speed $t^{-1}$.

Föllmer \& Schachermayer (2007) (Theorem 1.4) proved that if the stock price process has an average market price of risk above a threshold then asymptotic arbitrage holds. Using the LDP proved above, we first show that $S$ does not always admit an average market price of risk for $\gamma_{1}$ (Proposition 3.1) or $\gamma_{2}$ (Proposition 3.2) above any threshold. This is in particular so when the variance process is not ergodic $(b \leq 0)$. This however — as proved in Theorem 3.1 below — does not preclude absence of asymptotic arbitrage.

Proposition 3.1. Fix $\lambda \geq 0$ and $c>0$. The stock price process does not satisfy an average squared market price of risk $\gamma_{1}$ above the threshold $c$ with speed $t$ if either: (i) $b \leq 0$ or (ii) $b>0$ and $c>a \lambda^{2} / b$.

Proof. Note first that $\lambda=0$ implies $\gamma_{1} \equiv 0$ and hence $\mathbb{P}\left(t^{-1} \int_{0}^{t} \gamma_{1}^{2}(s) d s<c\right)=1$ for all $t>0$, so that the proposition is trivial. Assume from now on that $\lambda \neq 0$ and let $c$ be an arbitrary strictly positive real number. The definition of $\gamma_{1}$ in $(2.3)$ implies $\mathbb{P}\left(t^{-1} \int_{0}^{t} \gamma_{1}^{2}(s) d s \geq c\right)=\mathbb{P}\left(t^{-1} \int_{0}^{t} V_{s} d s \geq c / \lambda^{2}\right)=\mathbb{P}\left(t^{-1} X_{t}^{0,1,0} \geq c / \lambda^{2}\right)$. From Lemma 3.1 , the family $\left(t^{-1} X_{t}^{0,1,0}\right)_{t \geq 0}$ satisfies a LDP on $\mathbb{R}_{+}^{*}$ with rate function $\Lambda_{1,0}^{*}$. Hence

$$
\limsup _{t \uparrow \infty} \frac{1}{t} \log \mathbb{P}\left(X_{t}^{0,1,0} \geq \frac{c}{\lambda^{2}}\right) \leq-\inf _{\left\{x \geq c / \lambda^{2}\right\}} \Lambda_{1,0}^{*}(x) .
$$


When $b \leq 0$, $\inf _{\left\{x \geq c / \lambda^{2}\right\}} \Lambda_{1,0}^{*}(x)$ is strictly positive for all $c>0$. Thus $\mathbb{P}\left(t^{-1} X_{t}^{0,1,0} \geq c / \lambda^{2}\right)$ converges to zero as $t$ tends to infinity, which in turn implies that $\mathbb{P}\left(t^{-1} \int_{0}^{t} \gamma_{1}^{2}(s) d s<c\right)$ converges to 1 as $t$ tends to infinity, and statement (i) in the proposition follows. When $b>0$, consider the case $c>a \lambda^{2} / b$; then $\inf _{\left\{x \geq c / \lambda^{2}\right\}} \Lambda_{1,0}^{*}(x)$ is strictly positive and we end up with the same as in the case $b \leq 0$ which proves statement (ii) in the proposition.

Proposition 3.2. Fix $\lambda \geq 0$ and let $c>0$. The stock price process does not satisfy an average squared market price of risk $\gamma_{2}$ above the threshold $c$ with speed $t$ if any of the following conditions hold:

(1) $\lambda \rho(\mu-r)>0$;

(2) $\lambda \rho(\mu-r)<0$ and $c>-4 \lambda \rho(\mu-r) /\left(1-\rho^{2}\right)$;

(3) $\lambda \rho \neq 0, \mu=r$ and $b \leq 0$;

(4) $\lambda \rho \neq 0, \mu=r, b>0$ and $c>a \lambda^{4} \rho^{2} /\left(b\left(1-\rho^{2}\right)\right)$;

(5) $\lambda \rho=0$.

Remark 3.2. Note that the case $\lambda \rho=0$ precisely corresponds to the case of a complete market.

Proof. Let $c$ be an arbitrary strictly positive real number. Note first that if $\lambda \rho=0$, and $\mu=r$, then $\gamma_{2} \equiv 0$ and hence $\mathbb{P}\left(t^{-1} \int_{0}^{t} \gamma_{2}^{2}(s) d s<c\right)=1$ for all $t>0$. If $\mu \neq r$, then

$$
\mathbb{P}\left(\frac{1}{t} \int_{0}^{t} \gamma_{2}^{2}(s) d s \geq c\right)=\mathbb{P}\left(\frac{1}{t} \int_{0}^{t} \frac{d s}{V_{s}} \geq \frac{1-\rho^{2}}{(\mu-r)^{2}} c\right)=\mathbb{P}\left(\frac{X_{t}^{0,0,1}}{t} \geq \frac{1-\rho^{2}}{(\mu-r)^{2}} c\right),
$$

and Lemma A.1 implies that $\Lambda_{0,1}^{*}$ is strictly positive, so that (v) follows. Assume now that $\lambda \rho \neq 0$ and $\mu \neq r$. The definition of $\gamma_{2}$ in (2.3) implies that

$$
\begin{aligned}
\mathbb{P}\left(\frac{1}{t} \int_{0}^{t} \gamma_{2}^{2}(s) d s \geq c\right) \\
\quad=\mathbb{P}\left(\frac{(\mu-r)^{2}}{1-\rho^{2}} \frac{1}{t} \int_{0}^{t} \frac{d s}{V_{s}}+\frac{\lambda^{2} \rho^{2}}{1-\rho^{2}} \frac{1}{t} \int_{0}^{t} V_{s} d s \geq c+\frac{2 \rho \lambda(\mu-r)}{1-\rho^{2}}\right) \\
\quad=\mathbb{P}\left(\frac{X_{t}^{0, \beta, \delta}}{t} \geq c+\frac{2 \rho \lambda(\mu-r)}{1-\rho^{2}}\right),
\end{aligned}
$$

where $\beta=\frac{(\mu-r)^{2}}{1-\rho^{2}}>0, \delta=\frac{\lambda^{2} \rho^{2}}{1-\rho^{2}}>0$, and where $X^{0, \beta, \delta}$ is defined in (3.1). By Lemma 3.1 , the family $\left(X_{t}^{0, \beta, \delta} / t\right)_{t>0}$ satisfies a $\operatorname{LDP}$ on $(2 \sqrt{\delta \beta},+\infty)$ with rate function $\Lambda_{\beta, \delta}^{*}$, i.e.

$$
\limsup _{t \uparrow \infty} t^{-1} \log \mathbb{P}\left(\frac{1}{t} \int_{0}^{t} \gamma_{2}^{2}(s) d s \geq c\right) \leq-\inf \left\{\Lambda_{\beta, \delta}^{*}(x): x \geq c+\frac{2 \rho \lambda(\mu-r)}{1-\rho^{2}}\right\} .
$$

When $\lambda \rho(\mu-r)>0,\left[c+\frac{2 \rho \lambda(\mu-r)}{1-\rho^{2}},+\infty\right)$ is a subset of $(2 \sqrt{\beta \delta},+\infty)$, and (i) follows immediately from Lemma A.1. When $\lambda \rho(\mu-r)<0$, the interval $\left[c+\frac{2 \rho \lambda(\mu-r)}{1-\rho^{2}},+\infty\right)$ is 
a subset of $(2 \sqrt{\beta \delta},+\infty)$ if and only if $c>-\frac{4 \rho \lambda(\mu-r)}{1-\rho^{2}}>0$. Since $\beta \delta=\frac{\lambda^{2} \rho^{2}(\mu-r)^{2}}{\left(1-\rho^{2}\right)}>0$, Lemma A.1 implies that $\Lambda_{\beta, \delta}^{*}(x)>0$ for any $x>2 \sqrt{\beta \delta}=\frac{2|\lambda \rho(\mu-r)|}{1-\rho^{2}}$. Therefore, $\mathbb{P}\left(X_{t}^{0, \beta, \delta} / t \geq c+\frac{2 \rho \lambda(\mu-r)}{1-\rho^{2}}\right)$ converges to zero as $t$ tends to infinity. Then $\mathbb{P}\left(t^{-1} \int_{0}^{t} \gamma_{2}^{2}(s) d s<c\right)$ converges to one as $t$ tends to infinity. Assume that $\lambda \rho \neq 0$ and $\mu=r$. The definition of $\gamma_{2}$ in (2.3) implies that

$$
\mathbb{P}\left(\frac{1}{t} \int_{0}^{t} \gamma_{2}^{2}(s) d s \geq c\right)=\mathbb{P}\left(\frac{1}{t} \int_{0}^{t} V_{s} d s \geq \frac{1-\rho^{2}}{\lambda^{2} \rho^{2}} c\right)=\mathbb{P}\left(\frac{X_{t}^{0,1,0}}{t} \geq \frac{1-\rho^{2}}{\lambda^{2} \rho^{2}} c\right)
$$

and (iii) and (iv) then from Proposition 3.1.

We can now move on to our main theorem, which proves a partial arbitrage for the stock price process $S$.

Theorem 3.1. There exists $\gamma^{*}>0$ such that $S_{t}$ admits a partial $\left(e_{1}, 1 / 2\right)$-arbitrage for $t$ large enough, with

$$
e_{1}=\exp \left[\frac{\lambda V_{0}}{\sqrt{2 \sigma}}+\left(\frac{a \lambda}{\sqrt{2 \sigma}}+\gamma^{*}+\Lambda^{\beta}(1)\right) t\right], \text { for all } \lambda<-\frac{b}{\sqrt{2 \sigma}}-\frac{\gamma^{*} \sqrt{2 \sigma}}{2 a} .
$$

Remark 3.3. The threshold $e_{1}$ has the form $e_{1} \sim \mathrm{e}^{-\lambda_{1} t}$ for some $\lambda_{1}>0$, which links partial arbitrage to exponentially decaying failure probability characterized in Definition 2.3. We could slightly relax the constraint on $\lambda$, making it time dependent, because we only need to ensure that $e_{1} \in(0,1)$. Since we are only interested in large $t$, this is however not essential here. The sufficient condition on $\lambda$ is not necessary: for $\lambda=0$ and $\mu=r, Z_{t}=1$ almost surely for all $t \geq 0$, and $\mathbb{P}\left(Z_{t} \geq\right.$ $\left.\mathrm{e}^{-\gamma t}\right)=1$ for any $\gamma>0$.

Proof. Let $\gamma>0$ and define the set $A_{\lambda, t}:=\left\{Z_{t} \geq \mathrm{e}^{-\gamma t}\right\} \in \mathcal{F}_{t}$. Since the processes $W_{2}$ and $V$ are independent, the tower property for conditional expectation implies $\mathbb{E}\left(Z_{t}\right)=\mathbb{E}\left(\mathrm{e}^{-\int_{0}^{t} \gamma_{1}(s) d W_{1}(s)-\frac{1}{2} \int_{0}^{t} \gamma_{1}^{2}(s) d s}\right)$. Markov's inequality therefore yields

$$
\begin{aligned}
\mathbb{P}\left(A_{\lambda, t}\right) & \leq \frac{\mathbb{E}\left(Z_{t}\right)}{\exp (-\gamma t)}=\frac{\mathbb{E}\left[\exp \left(-\int_{0}^{t} \gamma_{1}(s) d W_{1}(s)-\frac{1}{2} \int_{0}^{t} \gamma_{1}^{2}(s) d s\right)\right]}{\mathrm{e}^{-\gamma t}} \\
& =\exp \left(\frac{\lambda V_{0}}{\sqrt{2 \sigma}}+\frac{a \lambda t}{\sqrt{2 \sigma}}+\gamma t\right) \mathbb{E}\left[\exp \left(-\frac{\lambda V_{t}}{\sqrt{2 \sigma}}-\left(\frac{b \lambda}{\sqrt{2 \sigma}}+\frac{\lambda^{2}}{2}\right) \int_{0}^{t} V_{s} d s\right)\right] \\
& =\exp \left[\frac{\lambda V_{0}}{\sqrt{2 \sigma}}+\left(\frac{a \lambda}{\sqrt{2 \sigma}}+\gamma\right) t\right] \Lambda_{t}^{\alpha, \beta}(t),
\end{aligned}
$$

where $\alpha=-\frac{\lambda}{\sqrt{2 \sigma}}$ and $\beta=-\frac{b \lambda}{\sqrt{2 \sigma}}-\frac{\lambda^{2}}{2}$. From the proof of Lemma 3.1, we know that $t^{-1} \log \Lambda_{t}^{\alpha, \beta}(t)$ converges to $\Lambda^{\beta}(1)$, which implies that for any $\eta>0$ there exists $\tilde{t}>0$ such that for any $t>\tilde{t}, \mathrm{e}^{\left(\Lambda^{\beta}(1)-\eta\right) t} \leq \Lambda_{t}^{\alpha, \beta}(t) \leq \mathrm{e}^{\left(\Lambda^{\beta}(1)+\eta\right) t}$. Therefore, for any 
$t>\tilde{t}$

$$
\mathbb{P}\left(Z_{t} \geq \mathrm{e}^{-\gamma t}\right) \leq \exp \left[\frac{\lambda V_{0}}{\sqrt{2 \sigma}}+\left(\frac{a \lambda}{\sqrt{2 \sigma}}+\gamma+\Lambda^{\alpha, \beta}(1)+\eta\right) t\right]
$$

Since $\eta$ can be chosen as small as desired, we simply need to prove that $\frac{a \lambda}{\sqrt{2 \sigma}}+\gamma+$ $\Lambda^{\beta}(1)<0$. From Appendix A, this inequality is satisfied whenever $\lambda<-\frac{b}{\sqrt{2 \sigma}}-$ $\frac{\gamma \sqrt{2 \sigma}}{2 a}$. Let $\varepsilon_{1}:=\exp \left[\frac{\lambda V_{0}}{\sqrt{2 \sigma}}+\left(\frac{a \lambda}{\sqrt{2 \sigma}}+\gamma+\Lambda^{\beta}(1)\right) t\right]$ and $\varepsilon_{2} \in(0,1)$. The (bounded and measurable) random variable $Y_{t}:=\varepsilon_{2} \mathbb{1}_{A_{\lambda, t}^{c}}-\varepsilon_{2} \frac{\mathbb{Q}\left(A_{\lambda, t}^{c}\right)}{\mathbb{Q}\left(A_{\lambda, t}\right)} \mathbb{1}_{A_{\lambda, t}}$ satisfies $\mathbb{E}_{\mathbb{Q}}\left(Y_{t}\right)=0$ for any $\mathbb{Q}$ in the space of absolutely continuous measures (with respect to $\mathbb{P}$ ) such that $S$ is a $\mathbb{Q}$-local martingale (in particular for all $\mathbb{Q} \in \mathcal{M}_{t}^{e}(S)$ ); hence there exists a measure $\widetilde{\mathbb{Q}}$, equivalent to $\mathbb{P}$ such that $Y$ is a $\widetilde{\mathbb{Q}}$-uniformly integrable martingale, and $Y_{t} \in \mathcal{K}_{t}$ by Theorem 9.5.2 in Delbaen \& Schachermayer (2006). ${ }^{\mathrm{a}}$ On $A_{\lambda, t}^{c}$, clearly $Y_{t}=\varepsilon_{2} \geq-\varepsilon_{2}$. Now, the family $\left(\left\{Z_{t} \geq \mathrm{e}^{-\gamma t}\right\}\right)_{\gamma>0}$ forms an increasing sequence of sets, and hence $\widetilde{\mathbb{Q}}\left(Z_{t} \geq \mathrm{e}^{-\gamma t}\right)$ is an increasing function of $\gamma$. Therefore there exists $\gamma^{*}>0$ such that for any $\gamma>\gamma^{*}, \widetilde{\mathbb{Q}}\left(A_{\lambda, t}^{c}\right) \leq \mathbb{Q}\left(A_{\lambda, t}\right)$, and therefore $Y_{t} \geq-\varepsilon_{2}$ on $A_{\lambda, t}$. This then yields $Y_{t} \geq-\varepsilon_{2}$ almost surely. Finally, since $\mathbb{P}\left(Y_{t}=\varepsilon_{2}\right)=$ $\mathbb{P}\left(A_{\lambda, t}^{c}\right) \geq 1-\varepsilon_{1}$, then $\mathbb{P}\left(Y_{t} \geq 1-\varepsilon_{2}\right) \geq \mathbb{P}\left(Y_{t}=\varepsilon_{2}\right) \geq 1-\varepsilon_{1}$ for $\varepsilon_{2} \in[1 / 2,1)$. Also, $\mathbb{P}\left(Y_{t}<1-\varepsilon_{2}\right)=\mathbb{P}\left(A_{\lambda, t}\right) \leq \varepsilon_{1}$, and hence $S$ allows for partial $\left(e_{1}, 1 / 2\right)$-arbitrage in the sense of Definition 2.2.

Remark 3.4. $\left(\varepsilon_{1}, \varepsilon_{2}\right)$-arbitrage in the sense of Definition 2.1 is harder to prove here since it would be equivalent to the existence of a set $A_{t} \in \mathcal{F}_{t}$, with $\mathbb{P}\left(A_{t}\right) \leq \varepsilon_{1}$ such that $\mathbb{Q}\left(A_{t}\right) \geq 1-\varepsilon_{2}$ holds for all $\mathbb{Q} \in \mathcal{M}^{e}(S)$ (by Proposition 2.1 in Föllmer $\&$ Schachermayer (2007)). Since the random variable $Z_{t}$ above depends on the parameter $\lambda$, the proof above shows that both $\mathbb{P}\left(A_{\lambda, t}\right) \leq \varepsilon_{1}$ and $\mathbb{Q}\left(A_{\lambda, t}\right) \geq 1-\varepsilon_{2}$ hold only when $\lambda<-\frac{b}{\sqrt{2 \sigma}}-\frac{\gamma \sqrt{2 \sigma}}{2 a}$; but the measure $\mathbb{Q}$ also depends on $\lambda$, and hence $\left(\varepsilon_{1}, \varepsilon_{2}\right)$-arbitrage may not hold.

\subsection{Case $t / f(t)$ and $f(t)$ both tend to infinity as t tends to infinity}

Let $b>0$, in which case the variance process is ergodic and its stationary distribution $\pi$ is a Gamma law with shape parameter $a / \sigma$ and scale parameter $\sigma / b$; namely $t^{-1} \int_{0}^{t} h\left(V_{s}\right) d s$ converges to $\int_{\mathbb{R}} h(x) \pi(d x)$ almost surely for any $h \in L^{1}(\pi)$ (see Kutoyants (2004)). In this section, we consider a continuous function $f: \mathbb{R}_{+}^{*} \rightarrow$ $\mathbb{R}_{+}$such that $t / f(t)$ tends to infinity as $t$ tends to infinity. We shall prove below that (under some conditions on the risk parameter $\lambda$ ) the ergodicity of the variance ensures that $S$ allows an asymptotic arbitrage with sublinear speed $f(t)$.

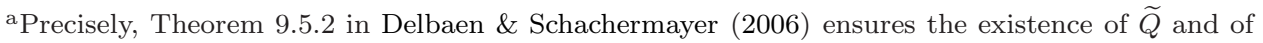
an initial endowment $\alpha$ such that $Y=\alpha+(H \cdot S)$. A simple no-arbitrage argument (similar to that in the finite probability space case, as in Corollary 2.2.8 in Delbaen \& Schachermayer (2006), see also the introduction of Delbaen \& Schachermayer (1998)) shows that here, $\alpha$ has to be equal to zero. 
Proposition 3.3. The stock price process $S$ in (2.1) has an average squared market price of risk $\gamma_{1}$ above the threshold $a \lambda^{2} / b$ with speed $f(t)$. If furthermore $a>\sigma$ and $\lambda \rho(\mu-r) \leq 0$, then there exists $c_{2}>0$ such that $S$ has an average squared market price of risk $\gamma_{2}$ above the threshold $c_{2}$ with speed $f(t)$.

Remark 3.5. As the proof shows, we can actually be more precise regarding the threshold $c_{2}$ :

- if $\mu=r$, then $c_{2}=\frac{a \lambda^{2} \rho^{2}}{b\left(1-\rho^{2}\right)}$;

- if $\mu \neq r$ and $\rho \lambda<0$, then no further condition on $c_{2}$ is needed;

- if $\mu \neq r$ and $\rho \lambda=0$, then $c_{2}=\frac{(\mu-r)^{2} b}{(a-\sigma)\left(1-\rho^{2}\right)}$.

It is rather interesting to compare this result with those of Proposition 3.1 and Proposition 3.2. Indeed, when $b>0$, if $f(t) \equiv t$ then the stock price process does not satisfy an average squared market price of risk $\gamma_{1}$ above the threshold $a \lambda^{2} / b$. However, when $t / f(t)$ tends to infinity, then $S$ has an average squared market price of risk $\gamma_{1}$ above the threshold $a \lambda^{2} / b$. When $b>0, \lambda \rho \neq 0$ and $\mu=r$, if $f(t) \equiv t$ then the stock price process does not satisfy an average squared market price of risk $\gamma_{2}$ above the threshold $\frac{a \lambda^{4} \rho^{2}}{b\left(1-\rho^{2}\right)}$, but does so above the threshold $\frac{a \lambda^{2} \rho^{2}}{b\left(1-\rho^{2}\right)}$ when $t / f(t)$ tends to infinity. Finally, when $b>0, \lambda \rho=0$ and $\mu \neq r$ the stock price process never satisfies an average squared market price of risk $\gamma_{2}$ with speed $f(t) \equiv t$, but does above the threshold $\frac{b(\mu-r)^{2}}{\left(1-\rho^{2}\right)(a-\sigma)}$ whenever $t / f(t)$ tends to infinity.

Proof of Proposition 3.3. Let $f$ be as stated in the proposition. For $b>0$, the variance process is ergodic and its stationary distribution is a Gamma law with shape parameter $a / \sigma$ and scale parameter $\sigma / b$ (see Kutoyants (2004)). In particular, $t^{-1} \int_{0}^{t} V_{s} d s$ converges in probability to $a / b$ as $t$ tends to infinity, and hence for any $c_{1} \in\left(0, a \lambda^{2} / b\right)$,

$$
\lim _{t \uparrow \infty} \mathbb{P}\left(\int_{0}^{t} \frac{\gamma_{1}^{2}(s)}{t} d s<c_{1}\right)=0, \quad \text { and hence } \quad \lim _{t \uparrow \infty} \mathbb{P}\left(\int_{0}^{t} \frac{\gamma_{1}^{2}(s)}{f(t)} d s<c_{1}\right)=0,
$$

which proves the first part of the proposition.

Consider now $\gamma_{2}$. When $\mu=r$, the definitions (2.3) imply that $\gamma_{2}=$ $-\rho \gamma_{1} / \sqrt{1-\rho^{2}}$, and hence

$$
\lim _{t \uparrow \infty} \mathbb{P}\left(\frac{1}{f(t)} \int_{0}^{t} \gamma_{2}^{2}(s) d s<c_{2}\right)=\lim _{t \uparrow \infty} \mathbb{P}\left(\frac{1}{f(t)} \int_{0}^{t} \gamma_{1}^{2}(s) d s<\frac{\left(1-\rho^{2}\right) c_{2}}{\rho^{2}}\right)
$$

is equal to zero if and only if $\left(1-\rho^{2}\right) c_{2} / \rho^{2} \in\left(0, a \lambda^{2} / b\right)$, and the proposition follows.

We now assume that $\mu \neq r$. If $a>\sigma$ we further know that (see Proposition 4 in Alaya \& Kebaier (2012)) $t^{-1} \int_{0}^{t} V_{s}^{-1} d s$ converges in probability to $b /(a-\sigma)$ as $t$ tends to infinity. Therefore for any $c \in(0, b /(a-\sigma))$ we have

$$
\lim _{t \uparrow \infty} \mathbb{P}\left(\frac{1}{t} \int_{0}^{t} \frac{d s}{V_{s}}<c\right)=0, \quad \text { and hence } \quad \lim _{t \uparrow \infty} \mathbb{P}\left(\frac{1}{f(t)} \int_{0}^{t} \frac{d s}{V_{s}}<c\right)=0 .
$$


Let $c_{2}, c_{1}^{\prime}, c_{2}^{\prime}$ be three strictly positive numbers such that $c_{2}=c_{1}^{\prime}+c_{2}^{\prime}$. The definition of $\gamma_{2}$ in (2.3) implies

$$
\begin{aligned}
\mathbb{P}\left(\frac{1}{f(t)} \int_{0}^{t} \gamma_{2}^{2}(s) d s<c_{2}\right) \\
=\mathbb{P}\left(\frac{1}{f(t)} \frac{(\mu-r)^{2}}{1-\rho^{2}} \int_{0}^{t} \frac{d s}{V_{s}}-\frac{2 \rho \lambda(\mu-r)}{1-\rho^{2}} \frac{t}{f(t)}+\frac{1}{f(t)} \frac{\lambda^{2} \rho^{2}}{1-\rho^{2}} \int_{0}^{t} V_{s} d s<c_{2}\right) \\
\leq \mathbb{P}\left(\frac{1}{f(t)} \frac{\lambda^{2} \rho^{2}}{1-\rho^{2}} \int_{0}^{t} V_{s} d s<c_{1}^{\prime}\right) \\
\quad+\mathbb{P}\left(\frac{1}{f(t)} \frac{(\mu-r)^{2}}{1-\rho^{2}} \int_{0}^{t} \frac{d s}{V_{s}}-\frac{2 \rho \lambda(\mu-r)}{1-\rho^{2}} \frac{t}{f(t)}<c_{2}^{\prime}\right) \\
=\mathbb{P}\left(\frac{1}{f(t)} \int_{0}^{t} \gamma_{1}^{2}(s) d s<c_{1}\right) \\
\quad+\mathbb{P}\left(\frac{1}{f(t)} \int_{0}^{t} \frac{d s}{V_{s}}<\frac{1-\rho^{2}}{(\mu-r)^{2}}\left[c_{2}^{\prime}+\frac{2 \rho \lambda(\mu-r)}{1-\rho^{2}} \frac{t}{f(t)}\right]\right),
\end{aligned}
$$

with $c_{1}^{\prime}=\frac{\rho^{2}}{1-\rho^{2}} c_{1}>0$. As long as $c_{1} \in\left(0, a \lambda^{2} / b\right)$, the first probability tends to zero as $t$ tends to infinity by (3.6). Now, when $\rho \lambda(\mu-r)<0$, then since $t / f(t)$ tends to infinity, the second probability tends to zero (as $t$ tends to infinity) by (3.7) because $c_{2}^{\prime}+\frac{2 \rho \lambda(\mu-r)}{1-\rho^{2}} \frac{t}{f(t)}$ tends to $-\infty$ (and because the variance process is non-negative almost surely). No condition on $c_{2}^{\prime}$ is needed here.

When $\rho \lambda=0$, then the first line of the equation above simplifies to

$$
\mathbb{P}\left(\frac{1}{f(t)} \int_{0}^{t} \gamma_{2}^{2}(s) d s<c_{2}\right)=\mathbb{P}\left(\frac{1}{f(t)} \frac{(\mu-r)^{2}}{1-\rho^{2}} \int_{0}^{t} \frac{d s}{V_{s}}<c_{2}\right) .
$$

From (3.7), it tends to zero as $t$ tends to infinity when $0<c_{2}<\frac{(\mu-r)^{2} b}{\left(1-\rho^{2}\right)(a-\sigma)}$, and hence the proposition follows from Definition 2.4.

We now state and prove our final result, namely a partial asymptotic arbitrage statement for the stock price process when the speed is sublinear.

Proposition 3.4. Fix $\gamma>0$. Then, for t large enough,

(1) if $\lambda \in \mathbb{R} \backslash[-\sqrt{2 b \gamma / a}, \sqrt{2 b \gamma / a}]$, then $S$ admits a partial $(0,1 / 2)$-arbitrage with speed $f(t)$;

(2) if $a>\sigma$ and $\lambda \rho(\mu-r) \leq 0$, then $S$ admits a partial $(0,1 / 2)$-arbitrage with speed $f(t)$,

- if and only if $\lambda \in \mathbb{R} \backslash\left[-\sqrt{\frac{2 b \gamma\left(1-\rho^{2}\right)}{a \rho^{2}}}, \sqrt{\frac{2 b \gamma\left(1-\rho^{2}\right)}{a \rho^{2}}}\right]$ when $\mu=r$ and $\rho^{2} \leq 1 / 2$;

- if and only if $\lambda \in \mathbb{R} \backslash[-\sqrt{2 b \gamma / a}, \sqrt{2 b \gamma / a}]$ when $\mu=r$ and $\rho^{2} \geq 1 / 2$;

- if $\mu \neq r$ and $\rho \lambda<0$;

- if $\mu \neq r, \rho \lambda=0$ and $\rho^{2}>1-\frac{(\mu-r)^{2} b}{2(a-\sigma) \gamma}$. 
Proof. Recall that we are in the framework of Proposition 3.3, so that $c_{1}>0$ and $c_{2}>0$ are the thresholds for $\gamma_{1}$ and $\gamma_{2}$ above which $S$ has an average squared market price of risk. In this proof, we follow steps similar to those in Föllmer \& Schachermayer (2007). For any $\varepsilon_{1}>0$, fix $0<\gamma<\bar{\gamma}<\frac{c_{1}}{2}=\frac{a \lambda^{2}}{2 b}$ and $t_{0}>$ $4 \bar{\gamma} /\left[(\bar{\gamma}-\gamma)^{2} \varepsilon_{1}\right]$ such that for any $t \geq t_{0}$ we have $\mathbb{P}\left(f(t)^{-1} \int_{0}^{t} \gamma_{1}^{2}(s) d s \leq 2 \bar{\gamma}\right)<\varepsilon_{1} / 2$. Define the stopping time $\tau_{1}:=t \wedge \inf \left\{s \in[0, t]: \int_{0}^{s} \gamma_{1}^{2}(u) d u \geq 2 \bar{\gamma} f(t)\right\}$. Let $\tilde{t}_{0}>t_{0}$ such that for any $t \geq t_{0}$ we have $f(t) \geq \tilde{t}_{0}$. Then for $t \geq \tilde{t}_{0}$ and using the fact that $\int_{0}^{\tau_{1}} \gamma_{1}^{2}(s) d s \leq 2 \bar{\gamma} f(t)$, Chebychev's inequality implies

$$
\mathbb{P}\left(\left|\int_{0}^{\tau_{1}} \gamma_{1}(s) d W_{1}(s)\right| \geq(\bar{\gamma}-\gamma) f(t)\right) \leq \frac{2 \bar{\gamma}}{(\bar{\gamma}-\gamma)^{2} f(t)}<\frac{\varepsilon_{1}}{2} .
$$

For $Z_{\tau_{1}}^{1}:=\exp \left(-\int_{0}^{\tau_{1}} \gamma_{1}(s) d W_{1}(s)-\frac{1}{2} \int_{0}^{\tau_{1}} \gamma_{1}^{2}(s) d s\right)$, we then obtain

$$
\begin{aligned}
\mathbb{P}\left(Z_{\tau_{1}}^{1} \geq \mathrm{e}^{-\gamma f(t)}\right)= & \mathbb{P}\left(-\int_{0}^{\tau_{1}} \gamma_{1}(s) d W_{1}(s)-\frac{1}{2} \gamma_{1}^{2}(s) d s \geq-\gamma f(t)\right) \\
\leq & \mathbb{P}\left(\left|\int_{0}^{\tau_{1}} \gamma_{1}(s) d W_{1}(s)\right| \geq(\bar{\gamma}-\gamma) f(t)\right) \\
& +\mathbb{P}\left(\int_{0}^{\tau_{1}} \frac{\gamma_{1}^{2}(s)}{2} d s \leq \bar{\gamma} f(t)\right) \\
\leq & \frac{\varepsilon_{1}}{2}+\frac{\varepsilon_{1}}{2}=\varepsilon_{1} .
\end{aligned}
$$

Let $A_{\lambda, t}:=\left\{Z_{\tau_{1}}^{1} \geq \mathrm{e}^{-\gamma f(t)}\right\} \in \mathcal{F}_{t}$. We obtain $\mathbb{P}\left(A_{\lambda, t}\right) \leq \varepsilon_{1}$. We are now in position to construct a contingent claim which satisfies the arbitrage estimates of the theorem. We (can) introduce the random variable $Y_{t}:=\varepsilon_{2} \mathbb{1}_{A_{\lambda, t}^{c}}-\varepsilon_{2} \frac{\mathbb{Q}\left(A_{\lambda, t}^{c}\right)}{\mathbb{Q}\left(A_{\lambda, t}\right)} \mathbb{1}_{A_{\lambda, t}}$ which satisfies the properties (i) and (ii) in Definition 2.2 with $e_{1}=0$ and $e_{2}=1 / 2$.

Assume now that $a>\sigma, \lambda \rho(\mu-r) \leq 0$, then $S$ has an average squared market price of risk $\gamma_{2}$ above a threshold $c_{2}>0$. For any $\varepsilon_{1}>0$, let $0<\gamma<\gamma^{\prime}<\frac{c_{2}}{2}$, and $t_{1}>\frac{4 \gamma^{\prime}}{\left(\gamma^{\prime}-\gamma\right)^{2} \varepsilon_{1}}$ such that, for $t \geq t_{1}, \mathbb{P}\left(f(t)^{-1} \int_{0}^{t} \gamma_{2}^{2}(s) d s \leq 2 \gamma^{\prime}\right)<\varepsilon_{1} / 2$. Define the stopping time $\tau_{2}$ by $\tau_{2}:=t \wedge \inf \left\{s \in[0, t]: \int_{0}^{s} \gamma_{2}^{2}(u) d u \geq 2 \gamma^{\prime} f(t)\right\}$ and the random variable $Z_{\tau_{2}}^{2}:=\exp \left(-f r m[o]--\int_{0}^{\tau_{2}} \gamma_{2}(s) d W_{2}(s)-\frac{1}{2} \int_{0}^{\tau_{2}} \gamma_{2}^{2}(s) d s\right)$. Let $\tilde{t}_{1}>t_{1}$ such that for any $t \geq t_{1}$ we have $f(t) \geq \tilde{t}_{1}$. Then for $t \geq \tilde{t}_{1}$, we have $\mathbb{P}\left(Z_{\tau_{2}}^{2} \geq \mathrm{e}^{-\gamma f(t)}\right) \leq$ $\varepsilon_{1}$. Let $B_{\lambda, t}:=\left\{Z_{\tau_{2}}^{2} \geq \mathrm{e}^{-\gamma f(t)}\right\} \in \mathcal{F}_{t}$. We obtain $\mathbb{P}\left(B_{\lambda, t}\right) \leq \varepsilon_{1}$. Similarly to the first case, we (can) introduce the random variable $Y_{t}:=\varepsilon_{2} \mathbb{1}_{B_{\lambda, t}^{c}}-\varepsilon_{2} \frac{\mathbb{Q}\left(B_{\lambda, t}^{c}\right)}{\mathbb{Q}\left(B_{\lambda, t}\right)} \mathbb{1}_{B_{\lambda, t}}$, and hence $S$ satisfies a partial $(0,1 / 2)$-arbitrage with speed $f(t)$.

Note that the constraint $a \lambda^{2} / b=c_{1}>2 \gamma$ reads $\lambda \in \mathbb{R} \backslash[-\sqrt{2 b \gamma / a}, \sqrt{2 b \gamma / a}]$. The constraints on $c_{2}$ depend on the sign of $\lambda \rho(\mu-r)$, as explained in Remark 3.5:

- if $\mu=r$ and $\rho^{2}<1 / 2$, then $c_{1}>c_{2}$; then $c_{2} / 2>\gamma$ if and only if $\lambda \in \mathbb{R} \backslash$ $\left[-\sqrt{\frac{2 b \gamma\left(1-\rho^{2}\right)}{a \rho^{2}}}, \sqrt{\frac{2 b \gamma\left(1-\rho^{2}\right)}{a \rho^{2}}}\right]$;

- if $\mu=r$ and $\rho^{2}>1 / 2$, then $c_{1}<c_{2}$; then $c_{1} / 2>\gamma$ if and only if $\lambda \in$ $\mathbb{R} \backslash[-\sqrt{2 b \gamma / a}, \sqrt{2 b \gamma / a}]$ 
- if $\mu \neq r$ and $\rho \lambda<0$, no further assumption on $\lambda$ is needed;

- if $\mu \neq r$ and $\rho \lambda=0$, then the constraint $0<\gamma<\frac{(\mu-r)^{2} b}{2(a-\sigma)\left(1-\rho^{2}\right)}$ has to hold.

\section{Acknowledgments}

The authors are deeply indebted to the two anonymous referees, whose comments and technical insights improved the quality of the present paper. AJ acknowledges financial support from the EPSRC First Grant EP/M008436/1.

\section{Appendix A. Large Deviations Results}

Proof of Lemma 3.1. Recall the standing assumption that $\beta$ and $\delta$ are never null simultaneously. We first prove the lemma in the case $b \neq 0$. The moment generating function of the random variable $X_{t}^{\alpha, \beta, \delta} / t$ is given by (see Proposition 2 in ?),

$$
\begin{aligned}
\Lambda_{t}(u)= & \mathbb{E}\left(\exp \left(\frac{\alpha u}{t} V_{t}+\frac{\beta u}{t} \int_{0}^{t} V_{s} d s+\frac{\delta u}{t} \int_{0}^{t} V_{s}^{-1} d s\right)\right) \\
= & \frac{\Gamma\left(\kappa+\frac{1}{2}(\nu+1)\right)}{\Gamma(\nu+1)} \exp \left\{\frac{b}{2 \sigma}\left(a t+V_{0}\right)-\frac{A V_{0}}{2 \sigma} \operatorname{coth}\left(\frac{A t}{2}\right)\right\} \\
& \times\left(\frac{A V_{0}}{2 \sigma \sinh (A t / 2)}\right)^{\frac{1}{2}(\nu+1)-\kappa} \\
& \times\left(\left(b-\frac{2 \sigma \alpha u}{t}\right) \frac{\sinh (A t / 2)}{A}+\cosh (A t / 2)\right)^{-\left(\kappa+\frac{1}{2}(\nu+1)\right)} \\
& \times{ }_{1} F_{1}\left(\kappa+\frac{\nu+1}{2}, \nu+1, \frac{A^{2} V_{0}}{2 \sigma \sinh (A t / 2)}\right)
\end{aligned}
$$

where $\kappa:=\frac{a}{2 \sigma}, A:=\sqrt{b^{2}-\frac{4 \sigma \beta u}{t}}, \nu:=\frac{1}{\sigma} \sqrt{(a-\sigma)^{2}-\frac{4 \sigma \delta u}{t}}$. Note that we are actually extending the result of Proposition 2 in ? here. Indeed, since characteristic functions can be extended in the complex plane up to the first singularity, the extension to positive values of $\alpha, \beta, \delta$ is trivial. The confluent hypergeometric function is defined by ${ }_{1} F_{1}(u, v, z)=\sum_{n \geq 0} \frac{u^{(n)}}{v^{(n)}} \frac{z^{n}}{n !}$, with $v^{(n)}$ denoting the rising factorial $v^{(n)}:=v(v+1) \ldots(v+n-1)$. As $t$ tends to infinity, $t^{-1} \log \left(\frac{\Gamma(\kappa+\nu / 2+1 / 2)}{\Gamma(\nu+1)}\right)$ clearly tends to zero and

$$
\begin{aligned}
\lim _{t \uparrow \infty} & \frac{1}{t} \log \left({ } _ { 1 } F _ { 1 } \left(\kappa+\frac{\nu+1}{2}, \nu+1,\right.\right. \\
& \left.\left.\frac{A^{2} V_{0}}{2 \sigma \sinh (A t / 2)[(b-2 \sigma \alpha u) \sinh (A t / 2)+\cosh (A t / 2)]}\right)\right)=0 .
\end{aligned}
$$


Therefore,

$$
\begin{aligned}
\Lambda^{\beta, \delta}(u):= & \lim _{t \uparrow \infty} t^{-1} \log \Lambda_{t}(t u) \\
= & \lim _{t \uparrow \infty} \frac{1}{t}\left\{\frac{b}{2 \sigma}\left(a t+V_{0}\right)-\frac{A V_{0}}{2 \sigma} \frac{\mathrm{e}^{A t / 2}+\mathrm{e}^{-A t / 2}}{\mathrm{e}^{A t / 2}-\mathrm{e}^{-A t / 2}}\right. \\
& +\left(\frac{\nu+1}{2}-\kappa\right) \log \left(\frac{A V_{0}}{\sigma\left(\mathrm{e}^{A t / 2}-\mathrm{e}^{-A t / 2}\right)}\right) \\
& \left.-\left(\kappa+\frac{\nu+1}{2}\right) \log \left(\frac{b-2 \sigma \alpha u}{A}\left(\frac{\mathrm{e}^{A t / 2}-\mathrm{e}^{-A t / 2}}{2}\right)+\frac{\mathrm{e}^{A t / 2}+\mathrm{e}^{-A t / 2}}{2}\right)\right\} \\
= & -\frac{\nu A}{2}-\frac{A}{2}+\frac{b a}{2 \sigma} \\
= & \frac{a b}{2 \sigma}-\frac{1}{2 \sigma} \sqrt{\left((a-\sigma)^{2}-4 \sigma \delta u\right)\left(b^{2}-4 \sigma \beta u\right)}-\frac{1}{2} \sqrt{b^{2}-4 \sigma \beta u},
\end{aligned}
$$

for $u \in \mathcal{D}_{\beta, \delta}$ where the interval $\mathcal{D}_{\beta, \delta}$ is given in (3.2). We can then immediately compute

$\partial_{u} \Lambda^{\beta, \delta}(u)=\frac{\sigma \beta}{\sqrt{b^{2}-4 \sigma \beta u}}-\frac{8 \sigma \delta \beta u-\beta(a-\sigma)^{2}-\delta b^{2}}{\sqrt{\left((a-\sigma)^{2}-4 \sigma \delta u\right)\left(b^{2}-4 \sigma \beta u\right)}}, \quad$ for any $u \in \mathcal{D}_{\beta, \delta}^{o}$,

and hence

$$
\partial_{u} \Lambda^{\beta, \delta}\left(\mathcal{D}_{\beta, \delta}^{o}\right)= \begin{cases}\mathbb{R}, & \text { if } \beta \delta \leq 0, \\ (2 \sqrt{\delta \beta},+\infty), & \text { if } \beta \geq 0, \quad \delta \geq 0, \\ (-\infty,-2 \sqrt{\delta \beta}), & \text { if } \beta \leq 0, \quad \delta \leq 0 .\end{cases}
$$

We also have, for any $u \in \mathcal{D}_{\beta, \delta}^{o}$,

$$
\partial_{u u} \Lambda^{\beta, \delta}(u)=\frac{2 \sigma^{2} \beta^{2}}{\left(b^{2}-4 \sigma \beta u\right)^{3 / 2}}+\frac{2 \sigma\left(\delta b^{2}-\beta(a-\sigma)^{2}\right)^{2}}{\left(\left((a-\sigma)^{2}-4 \sigma \delta u\right)\left(b^{2}-4 \sigma \beta u\right)\right)^{3 / 2}} .
$$

Therefore $\Lambda^{\beta, \delta}$ is strictly convex on $\mathcal{D}_{\beta, \delta}$, and the Gärtner-Ellis theorem (see Dembo $\&$ Zeitouni (1993)) only applies on subsets of $\partial_{u} \Lambda^{\beta, \delta}\left(\mathcal{D}_{\beta, \delta}^{o}\right)$. For any $x \in$ $\partial_{u} \Lambda^{\beta, \delta}\left(\mathcal{D}_{\beta, \delta}^{o}\right)$, the equation $\partial_{u} \Lambda^{\beta, \delta}(u)=x$ has a unique solution $u^{*}(x)$ and hence $\Lambda_{\beta, \delta}^{*}(x):=\sup _{u \in \mathcal{D}_{\beta, \delta}}\left\{u x-\Lambda^{\beta, \delta}(u)\right\}=u^{*}(x) x-\Lambda^{\beta, \delta}\left(u^{*}(x)\right)$.

We now move on to the case $b=0$. From Corollary 1 in ?, the moment generating function of the random variable $X_{t}^{\alpha, \beta, \delta}$ is given by

$$
\begin{aligned}
\Lambda_{t}(u) & =\mathbb{E}\left[\exp \left(\frac{\alpha u}{t} V_{t}+\frac{\beta u}{t} \int_{0}^{t} V_{s} d s+\frac{\delta u}{t} \int_{0}^{t} V_{s}^{-1} d s\right)\right] \\
& =\frac{\Gamma\left(\kappa+\frac{1}{2}(\nu+1)\right)}{\Gamma(\nu+1)}\left(\frac{\zeta_{u} V_{0}}{\sigma \sinh \left(\zeta_{u} t\right)}\right)^{\frac{1}{2}(\nu+1)-\kappa}
\end{aligned}
$$




$$
\begin{aligned}
& \times\left[\frac{-\sqrt{\sigma} \alpha u / t}{\sqrt{-\beta u / t}} \sinh \left(\zeta_{u} t\right)+\cosh \left(\zeta_{u} t\right)\right]^{-\left(\kappa+\frac{1}{2}(\nu+1)\right)} \\
& \times \mathrm{e}_{1}^{-\frac{V_{0} \zeta_{u}}{\sigma} \operatorname{coth}\left(\zeta_{u} t\right)} \\
& \times F_{1}\left(\kappa+\frac{\nu+1}{2}, \nu+1, \frac{V_{0} \zeta_{u}}{\sigma \sinh \left(\zeta_{u} t\right)\left(-\frac{\sigma \alpha u}{\zeta_{u} t} \sinh \left(\zeta_{u} t\right)+\cosh \left(\zeta_{u} t\right)\right)}\right),
\end{aligned}
$$

where $\kappa:=\frac{a}{2 \sigma}$, and $\nu:=\frac{1}{\sigma} \sqrt{(a-\sigma)^{2}-\frac{4 \sigma \delta u}{t}}$, and $\zeta_{u}:=\sqrt{-\frac{\sigma \beta u}{t}}$. Similar to the case $b \neq 0$, we obtain

$$
\begin{aligned}
& \lim _{t \uparrow \infty} \frac{1}{t} \log _{1} \\
& \quad \times F_{1}\left(\begin{array}{c}
\kappa+\frac{\nu+1}{2}, \nu+1, \frac{\sqrt{-\sigma \beta} V_{0}}{\sigma \sinh (\sqrt{-\sigma \beta} u)} \\
\left(-\frac{\sqrt{\sigma} \alpha}{\sqrt{-\beta}} \sinh (\sqrt{-\sigma \beta} u)+\cosh (\sqrt{-\sigma \beta} u)\right)
\end{array}\right)=0 .
\end{aligned}
$$

Therefore, the limiting cumulant generating function of $X_{t}^{\alpha, \beta, \delta}$ reads

$$
\begin{aligned}
\Lambda^{\beta, \delta}(u):= & \lim _{t \uparrow \infty} t^{-1} \log \Lambda_{t}(t u) \\
= & \lim _{t \uparrow \infty} \frac{1}{t}\left\{-\frac{\xi_{u} V_{0}}{\sigma} \frac{\mathrm{e}^{\xi_{u} t}+\mathrm{e}^{-\xi_{u} t}}{\mathrm{e}^{\xi_{u} t}-\mathrm{e}^{-\xi_{u} t}}+\left(\frac{\nu+1}{2}-\kappa\right) \log \left(\frac{2 \xi_{u} V_{0}}{\sigma\left(\mathrm{e}^{\xi_{u} t}-\mathrm{e}^{-\xi_{u} t}\right)}\right)\right. \\
& \left.-\left(\kappa+\frac{\nu+1}{2}\right) \log \left[-\frac{\sqrt{\sigma} \alpha u}{\sqrt{-\beta u}}\left(\frac{\mathrm{e}^{\xi_{u} t}-\mathrm{e}^{-\xi_{u} t}}{2}\right)+\frac{\mathrm{e}^{\xi_{u} t}+\mathrm{e}^{-\xi_{u} t}}{2}\right]\right\} \\
= & -\xi_{u}-\frac{1}{\sigma} \xi_{u} \sqrt{(a-\sigma)^{2}-4 \sigma \delta u},
\end{aligned}
$$

with $\xi_{u}:=\sqrt{-\sigma \beta u}$, for any $u \in \mathcal{D}_{\beta, \delta}$ where this interval now reads

$$
\mathcal{D}_{\beta, \delta}= \begin{cases}{\left[0, \frac{(a-\sigma)^{2}}{4 \sigma \delta}\right],} & \text { if } \beta \leq 0 \text { and } \delta \geq 0, \\ {\left[\frac{(a-\sigma)^{2}}{4 \sigma \delta}, 0\right],} & \text { if } \beta \geq 0 \text { and } \delta \leq 0, \\ \mathbb{R}_{-}, & \text {if } \beta \geq 0 \text { and } \delta \geq 0, \\ \mathbb{R}_{+}, & \text {if } \beta \leq 0 \text { and } \delta \leq 0,\end{cases}
$$

where again we use the convention that $1 /|\beta|=+\infty$ when $\beta=0$. Then

$$
\begin{aligned}
\partial_{u} \Lambda^{\beta, \delta}(u)= & \frac{\sigma \beta}{2 \sqrt{-\sigma \beta u}}+\frac{\beta \sqrt{(a-\sigma)^{2}-4 \sigma \delta u}}{2 \sqrt{-\sigma \beta u}} \\
& +\frac{2 \delta \sqrt{-\sigma \beta u}}{\sqrt{(a-\sigma)^{2}-4 \sigma \delta u}}, \quad \text { for any } u \in \mathcal{D}_{\beta, \delta}^{o},
\end{aligned}
$$


and hence

$$
\partial_{u} \Lambda^{\beta, \delta}\left(\mathcal{D}_{\beta, \delta}^{o}\right)= \begin{cases}\mathbb{R}, & \text { if } \beta \delta \leq 0 \\ (2 \sqrt{\delta \beta},+\infty), & \text { if } \beta \geq 0 \text { and } \delta \geq 0 \\ (-\infty,-2 \sqrt{\delta \beta}), & \text { if } \beta \leq 0 \text { and } \delta \leq 0\end{cases}
$$

We also have

$$
\begin{aligned}
\partial_{u u} \Lambda^{\beta, \delta}(u)= & \frac{\sigma^{2} \beta^{2}}{4(-\sigma \beta u)^{3 / 2}}-\frac{\beta(a-\sigma)^{2}}{4 u \sqrt{(a-\sigma)^{2}-4 \sigma \delta u} \sqrt{-\sigma \beta u}} \\
& -\frac{\sigma \beta \delta(a-\sigma)^{2}}{\left((a-\sigma)^{2}-4 \sigma \delta u\right)^{3 / 2} \sqrt{-\sigma \beta u}} .
\end{aligned}
$$

Clearly then, $\Lambda^{\beta, \delta}$ is convex on $\mathcal{D}_{\beta, \delta}$, and the Gärtner-Ellis theorem only applies on subsets of $\partial_{u} \Lambda^{\beta, \delta}\left(\mathcal{D}_{\beta, \delta}^{o}\right)$. For any $x \in \partial_{u} \Lambda^{\beta, \delta}\left(\mathcal{D}_{\beta, \delta}^{o}\right)$, the equation $\partial_{u} \Lambda^{\beta, \delta}(u)=x$ has a unique solution $u^{*}(x)$ and hence $\Lambda_{\beta, \delta}^{*}(x):=\sup _{u \in \mathcal{D}_{\beta, \delta}}\left\{u x-\Lambda^{\beta, \delta}(u)\right\}=u^{*}(x) x-$ $\Lambda_{\beta, \delta}\left(u^{*}(x)\right)$, and the lemma follows.

Lemma A.1. For any $x \in \partial_{u} \Lambda^{\beta, \delta}\left(\mathcal{D}_{\beta, \delta}^{o}\right)$, the equation $\partial_{u} \Lambda^{\beta, \delta}\left(u^{*}(x)\right)=x$ admits a unique solution $u^{*}(x) \in \mathcal{D}_{\beta, \delta}^{o}$. The function $\Lambda_{\beta, \delta}^{*}$ is strictly convex and satisfies $\Lambda_{\beta, \delta}^{*}(x)=u^{*}(x) x-\Lambda^{\beta, \delta}\left(u^{*}(x)\right)$ on $\partial_{u} \Lambda^{\beta, \delta}\left(\mathcal{D}_{\beta, \delta}^{o}\right)$ and is (positive) infinite outside. In the case $\beta \delta \geq 0, \Lambda_{\beta, \delta}^{*}$ is strictly positive. When $\beta \delta \leq 0, \Lambda_{\beta, \delta}^{*}$ admits a unique minimum, which is equal to zero (and is attained at the origin) if and only if $a>\sigma$.

Proof. When $\beta \delta<0$, the image of $\mathcal{D}_{\beta, \delta}^{o}$ by $\partial_{u} \Lambda^{\beta, \delta}$ is the whole real line, and the representation of $\Lambda_{\beta, \delta}^{*}$ in the lemma clearly follows. Now, suppose there exists $\bar{x} \in \mathbb{R}$ such that $\Lambda_{\beta, \delta}^{*}(\bar{x})=0$. Then there exists some (possibly nonunique) $u^{*}(\bar{x}) \in \mathcal{D}_{\beta, \delta}$ such that $u^{*}(\bar{x}) \bar{x}=\Lambda^{\beta, \delta}\left(u^{*}(\bar{x})\right)$, i.e. $\Lambda^{\beta, \delta}\left(u^{*}(\bar{x})\right) / u^{*}(\bar{x})=\bar{x}$. But $u^{*}(\bar{x})$ also satisfies $\partial_{u} \Lambda^{\beta, \delta}\left(u^{*}(\bar{x})\right)=\bar{x}$. A straightforward analysis shows that the equality $\partial_{u} \Lambda^{\beta, \delta}(u)=$ $\Lambda^{\beta, \delta}(u) / u$ is satisfied if and only if $u=0$ and $a>\sigma$.

When $\beta>0$ and $\delta>0$, for any $x \leq 2 \sqrt{\beta \delta}$, the map $u \mapsto u x-\Lambda^{\beta, \delta}(u)$ is strictly decreasing on $\mathcal{D}_{\beta, \delta}^{o}$, and the result follows. By definition, the function $\Lambda_{\beta, \delta}^{*}$ admits a (unique) minimum $\bar{x}$ if and only if (i) there exists $u(\bar{x}) \in \mathcal{D}_{\beta, \delta}$ such that $u(\bar{x}) \bar{x}=\Lambda^{\beta, \delta}(u(\bar{x}))$ and (ii) $\Lambda^{\beta, \delta}(u)>u \bar{x}$ for any $u \in \mathcal{D}_{\beta, \delta} \backslash\{u(\bar{x})\}$. A straightforward analysis shows that the function $u \mapsto \Lambda^{\beta, \delta}(u) / u$ on $\mathbb{R}_{-}^{*}$ is strictly increasing and maps $\mathbb{R}_{-}^{*}$ to $(2 \sqrt{\beta \delta},+\infty)$. On $\mathbb{R}_{+}^{*} \cap \mathcal{D}_{\beta, \delta}$, it is strictly increasing and maps this interval to $(-\infty,-2 \sqrt{\beta \delta})$. Therefore, the inequality $\Lambda(u)>u x$ holds if and only if both (a) $\Lambda^{\beta, \delta}(u) / u>x$ for $u \in \mathbb{R}_{+}^{*} \cap \mathcal{D}_{\beta, \delta}$ and (b) $\Lambda^{\beta, \delta}(u) / u<x$ for $u<0$. Case (b) clearly only holds for $x<2 \sqrt{\beta \delta}$, which is not valid. The other cases are treated analogously.

\section{References}

M. B. Alaya \& A. Kebaier (2012) Parameter estimation for the square root diffusions: Ergodic and non ergodic case, Stochastic Models 28 (4), 609-634. 
M. M. Bidima \& M. Rasonyi (2012) On long-term arbitrage opportunities in Markovian models of financial markets, Annals of Operations Research 200 (1), 131-146.

M. M. Bidima \& M. Rasonyi (2015) Asymptotic exponential arbitrage and utility-based asymptotic arbitrage in Markovian models of financial markets, Acta Applicandae Mathematicae 138 (1), 1-15.

F. Cordero \& L. Perez-Ostafe (2015) Strong asymptotic arbitrage in the large fractional binary market, Preprint, http://arXiv.org/abs/1501.07445.

F. Delbaen \& W. Schachermayer (1998) A simple counter-example to several problems in the theory of asset pricing, Mathematical Finance 8 (1), 1-11.

F. Delbaen \& W. Schachermayer (2006) The Mathematics of Arbitrage. Berlin: Springer Finance.

A. Dembo \& O. Zeitouni (1993) Large Deviations Techniques and Applications. Boston: Jones and Bartlet Publishers.

K. Du \& A. Neufeld (2013) A note on asymptotic exponential arbitrage with exponentially decaying failure probability, Journal of Applied Probability 50 (3), 801-809.

H. Föllmer \& W. Schachermayer (2007) Asymptotic arbitrage and large deviations, Mathematics and Financial Economics 1 (34), 213-249.

J. P. Fouque, G. Papanicolaou, R. Sircar \& K. Solna (2011) Multiscale Stochastic Volatility for Equity, Interest Rate, and Credit Derivatives. Cambridge, UK: Cambridge University Press.

J. Gatheral (2006) The Volatility Surface: A Practitioner's Guide. New Jersey: Wiley.

S. Heston (1993) A closed-form solution for options with stochastic volatility with applications to bond and currency options, The Review of Financial Studies 6, 327-342.

C. C. Heyde \& B. Wong (2006) On changes of measure in stochastic volatility models, Journal of Applied Mathematics and Stochastic Analysis 2006, 18130.

M. Jeanblanc, M. Yor \& M. Chesney (2009) Mathematical Methods for Financial Markets. London: Springer Finance.

Y. Kabanov \& D. Kramkov (1998) Asymptotic arbitrage in large financial markets, Finance and Stochastics 2, 143-172.

I. Klein, E. Lépinette \& L. Perez-Ostafe (2014) Asymptotic arbitrage with small transaction costs, Finance and Stochastics 18 (4), 917-939.

I. Klein \& W. Schachermayer (1996) Asymptotic arbitrage in non-complete large financial markets, Theory of Probability and Its Applications 41 (4), 927-934.

Y. A. Kutoyants (2004) Statistical Inference for Ergodic Diffusion Processes. London: Springer-Verlag.

D. B. Rokhlin (2008) Asymptotic arbitrage and numéraire portfolio in large financial markets, Finance and Stochastics 12 (2), 173-194. 